\title{
Significance of SODD expression in childhood acute lymphoblastic leukemia and its influence on chemotherapy
}

H.F. Tao, Y.S. Liu, J.L. Fang, Y.Z. Su, F.H. Chen, L.Y. Zhou and Y.S. Zhu

Department of Hematology,

The First Affiliated Hospital of Shantou University Medical College,

Shantou, China

Corresponding author: Y.S. Liu

E-mail: yuanshengliucn@126.com

Genet. Mol. Res. 13 (1): 2020-2031 (2014)

Received January 4, 2013

Accepted June 14, 2013

Published March 24, 2014

DOI http://dx.doi.org/10.4238/2014.March.24.6

ABSTRACT. This study explored the clinical significance of silencer
of death domain (SODD) expression in childhood acute lymphoblastic
leukemia (ALL) and its influence on chemotherapy as well as the
effect of SODD expression on apoptosis of leukemic cells. The
expression of SODD proteins in different ALL groups was determined
by immunocytochemistry. The SODD RNAi-interfering plasmid was
constructed and transferred to Jurkat cells, and the effects of SODD
expression on cell proliferation and apoptosis were analyzed using the
MTT and FCM methods. The expressions of SODD, Phospho-NF-
кB-P65, Bcl-2, and Caspase 3 were detected by Western blot analysis.
The expression of SODD proteins was significantly higher in the ALL
groups than in the control group (P $<0.05)$. The positive expression rate
of SODD was significantly higher in refractory/relapsed and clinical
high-risk groups than in standard-risk, initial treatment, and complete
remission groups (P < 0.05 ). Microtubule-targeting drugs such as
vincristine and taxol can notably down-regulate SODD expression
during apoptosis, whereas DNR, and Ara-c cannot. The sensitivity of 
Jurkat cells to chemotherapeutic drugs increased with down-regulated SODD expression induced by SODD-interfering plasmid transfection. The sensitivity of the cells transfected with SODD-cloning genes decreased. SODD expression was high in the ALL children. These findings indicated that SODD over-expression might be correlated with the clinical classification, curative effect, and prognosis of ALL cells. Microtubule-targeting drugs can specifically down-regulate SODD expression in leukemic cells, thereby increasing the sensitivity of leukemic cells to SODD-targeting chemotherapeutics. In contrast, increased SODD expression tends to reduce sensitivity.

Key words: Silencer of death domain (SODD); Chemotherapeutic; Childhood acute lymphoblastic leukemia; Apoptosis of leukemic cells

\section{INTRODUCTION}

Acute lymphocytic leukemia (ALL) is the most common type of childhood leukemia. To date, chemotherapy is the major treatment method for ALL. Relatively high remission cure rates can be obtained clinically by chemotherapy, but $30 \%$ of cases respond poorly. Of the cases that respond poorly, drug resistance is the main cause of treatment failure (Dluzniewska et al., 2010). Drug resistance can be attributed to the administration of anti-leukemia drugs or by gene defects in the tumor cells as confirmed in the initial diagnosis (Cwiklińska et al., 2010; Czech et al., 2011). The abnormal expression of some apoptosis-related factors is the basic cause of the abnormal apoptosis of tumor cells. The induced expression differences result in associations with chemosensitivity as well as the drug resistance of tumor cells, which is the basis of chemotherapeutic effect evaluation (Xi et al., 2011). Therefore, an examination of the role played by apoptosis-related factors in the apoptotic evasion of leukemic cells, and the search for the malignant biomarker of tumor cells (i.e., the target) to which molecule-targeting gene therapy is specifically given, are of great significance. In particular, these types of studies can facilitate the reversal of tumor cell drug resistance, promote increased remission, and cure rates of refractory leukemia.

Silencer of death domain (SODD) is a newly discovered apoptosis-regulating gene located at $8 \mathrm{q} 12$. As a member of the BAG protein family, SODD is also named BAG-4 and is seen widely in the cytoplasm of many cells (Elstrand et al., 2009). With specific integration into the death domain (DD) of the TNFR-I, HSP/HSC70, DR3, and Bcl-2 cytoplasmic segments, SODD is currently considered a key negative-control protein in the apoptosis-inducing signaling pathway mediated by TNFR-I. SODD plays important roles in the abnormal proliferation and apoptotic evasion of tumor cells (Tao et al., 2007a,b). However, to the best of our knowledge, whether the over-expression of SODD contributes to the initiation and development of childhood ALL remains to be elucidated. Therefore, to explore the clinical significance of the over-expression of SODD in childhood ALL and to improve the chemotherapeutic effect on ALL, we clinically detected the expression of SODD in the bone marrow cells of children with ALL. We also constructed a small SODD-interfering plasmid and SODD sub-cloning genes. Subsequently, these were transfected into leukemic cells to investigate the different effects of enhanced and inhibited SODD expression on the apoptotic sensitivity of leukemic cells to chemotherapeutic drugs. Further, we conducted a screening for specific 
drugs of SODD with the expectation of providing some theoretical and experimental grounds for treatment with anti-apoptosis molecules.

\section{MATERIAL AND METHODS}

\section{Samples}

From March 2004 to March 2006, 107 children with ALL underwent treatment at Tongji Hospital. Their ages ranged from 10 months to 14 years $(6.2 \pm 5$ years $)$. ALL children were divided into initial treatment (51 cases), refractory/relapsed (16 cases), and complete remission (CR) (40 cases) groups. The initial treatment group was further sub-grouped into standard-risk (32 cases) and high-risk (HR, 19 cases) groups. Twenty-six children with nonhematologic malignant diseases constituted the control group. Their ages ranged from 1-12 years $(7+6$ years $)$. Among the 26 children in the control group, 15 suffered from ITP, 6 from iron deficiency anemia, and 5 from hyperplastic anemia. The diagnosis in all cases was confirmed by simultaneous morphological, immunological, and cytological examination. The bone marrow cells of the initial treatment group were collected before chemotherapy and at the end of the second week during remission-inducing chemotherapy. For the refractory/relapsed and CR groups, the bone marrow cells were collected when necessary. After completion of cell slide preparation, the cells were kept in a refrigerator at $-20^{\circ} \mathrm{C}$. This study was conducted in accordance with the declaration of Helsinki, with approval from the Ethics Committee of The First Affiliated Hospital of Shantou University Medical College (Permission No. 20080828001). Written informed consent was obtained from all parents or guardians.

\section{Cell culture}

Human acute T-ALL Jurkat cells were used for in vitro experiments. These cells were grown in RPMI1640 medium and supplemented with $10 \%$ fetal calf serum in $5 \% \mathrm{CO}_{2}$ at $37^{\circ} \mathrm{C}$ in $95 \%$ relative humidity incubator. Chemotherapeutic drugs (VCR, $1 \mu \mathrm{g} / \mathrm{mL}$; PTX, $100 \mathrm{ng} / \mathrm{mL}$; Dexa, $1 \mu \mathrm{g} / \mathrm{mL}$; DNR, $0.5 \mu \mathrm{g} / \mathrm{mL}$; MTX, $0.5 \mathrm{~g} / \mathrm{mL}$; Ara-c, $0.5 \mu \mathrm{g} / \mathrm{mL}$; and LASP, $1 \mathrm{U} / \mathrm{mL}$ ) were applied to the Jurkat cells during the logarithmic growth phase. Samples from the different groups were harvested at set time points.

\section{SODD expression}

The bone marrow slides were immobilized in cold $\left(4^{\circ} \mathrm{C}\right)$ acetone. The working concentration of the SODD polyclonal antibodies was a 1:100 dilution. All procedures were performed according to kit instructions. The samples were stained by hematoxylin and mounted by neutral gum. Phosphate-buffered saline (PBS) was used to replace the primary antibody as a negative control. The cell-staining intensity and positive rate were measured with an HPLAS-1000 analysis system, which provided reports with both graphics and text. At a high magnification, 200 leukemic cells of each sample were observed randomly. SODD-positive cell numbers $<10 \%$ were considered to be negative expression, whereas positive cell numbers $\geq 10 \%$ were considered to be positive expression. 


\section{Construction of the shRNA vector}

Based on the SODD sequence (GenBank: NM_004874), 3 micro-molecular interfering sequences were designed:

1) BAG41 742 GCTTATTATGCACCTGGTTAT 38.095\%

2) BAG42 1229 GCAGTGTCCATCAGTACGAAT $47.619 \%$

3) BAG43 1606 GTTTGTAAGATTCAGGCCATA $38.095 \%$

DNA template-based primers were designed and synthesized. Primer structure: Eco31I + Sense + Loop + Antisense + Termination signal + SacI $+E c o 31 I$. The multiple cloning sites of pGenesil1.1, pGenesil1.2, and pGenesil1.3 were as follows:

1) MluI-hU6 promoter-Insert DNA-SacI-HindIII-BamHI-EcoRI-SalI

2) SalI-EcoRI-BamHI-mU6 promoter-Insert DNA-SacI-HindIII-MluI

3) MluI-HindIII-BamHI-h7SK promoter-Insert DNA-SacI-EcoRI-SalI

The 3 plasmids were digested completely by Eco31I. The target fragments were recovered by $1 \%$ agarose gel electrophoresis to obtain the linear vectors (for detailed experimental procedures, refer to molecular cloning). Enzyme analysis and gene sequencing results showed that the cloned plasmids were inserted properly and that their quality met the design.

\section{Construction of the SODD expression vector}

The PCR primers of the SODD sub-cloning gene were as follows: (P1) 5'-CCGCTC GAGGCCACCATGTCGGCCCTGAGGCGCTCGGGCT-3'; (P2) 5'-CGGGATCCTAATCC TTTTTTTTCTAATTTTTCC-3'.

Under the amplification conditions, an initial pre-denaturation step was conducted for $5 \mathrm{~min}$ at $94^{\circ} \mathrm{C}$. This was followed by 30 cycles at $94^{\circ} \mathrm{C}$ for $20 \mathrm{~s}, 55.2^{\circ} \mathrm{C}$ for $25 \mathrm{~s}$, and $72^{\circ} \mathrm{C}$ for $1 \mathrm{~min}$ and $20 \mathrm{~s}$. The final cycle was performed at $72^{\circ} \mathrm{C}$ for $3 \mathrm{~min}$. The SODD genes were amplified by PCR. The PCR products were sequenced after ligation into the T vector. SODD was sub-cloned into the PEGFP-C1 vector. TS-BAG4 was digested by BamHI/XhoI and then ligated with PEGFP-C1 that had been digested with $B a m \mathrm{HI} / X h o \mathrm{I}$. The ligated products were then transformed into Escherichia coli DH5a. Clones were also selected and identified. These clones were confirmed by BglII enzyme digestion and gene sequencing. The clone was named PEGFP-C1-SODD.

\section{Cell transfection}

Transfection of plasmids was conducted in Jurkat cells in logarithmic phase using cationic liposome Lipofectamine 2000. The cells were inoculated on a 24-well plate at a density of $5 \times 10^{5} /$ well and cultured overnight. Two types of solutions were prepared in sterile tubes: 1) Solution A, $100 \mu \mathrm{L}$ RPMI1640 medium containing $2 \mu \mathrm{g}$ pre-transfection plasmids without serum or antibiotics; and 2) Solution B, $100 \mu \mathrm{L}$ RPMI1640 medium containing $2 \mu \mathrm{L}$ liposomes without serum or antibiotics (incubated at room temperature for $25 \mathrm{~min}$ after the addition of liposomes). Solutions A and B were mixed and then incubated at room temperature for $15 \mathrm{~min}$. The mixture $(100 \mu \mathrm{L})$ was transferred gently to the cell surface to cover the cells for the 6 -h culture. The transfection liquid was removed, and cell culture liquid $(200 \mu \mathrm{L})$ was added for a 48-h culture to observe EGFP expression. 


\section{Western blot analysis}

Drug-treated and transfected cells were scraped off, and total protein was extracted. After denaturation, the protein sample was fractionated on SDS-PAGE at a load of $50 \mu \mathrm{g} /$ well and then electro-transferred to a PVDF membrane. The membrane was treated with $0.1 \%$ TBS-T solution containing 5\% skimmed milk at room temperature for $2 \mathrm{~h}$ and incubated with primary antibodies at $4^{\circ} \mathrm{C}$ overnight. After TBS-T washing (10 min for five times), the mixture was incubated with horseradish peroxidase-conjugated anti-rabbit or anti-mouse antibodies at room temperature and oscillated for $2 \mathrm{~h}$. After washing with TBS-T (10 min for five times), the membrane was developed using chemiluminescence. The integrated optical density was quantitated by the Leica Q Win image analysis software using the SODD/beta-actin ratio to determine the SODD expression level and the Bcl-2/beta-actin ratio for the Bcl-2 expression level. The mean value was calculated from 3 independent experiments.

\section{Flow cytometry (FCM)}

To discard the medium, $1 \times 10^{6}$ cells in each sample group were harvested and centrifuged. After washing twice in PBS, the cells were resuspended in $400 \mu \mathrm{L}$ binding buffer. The cell suspension was mixed with $5 \mu \mathrm{L}$ Annexin V-FITC and $5 \mu \mathrm{L}$ propidium iodide and incubated at room temperature for 5-15 min. Apoptosis was observed and measured with a fluorescence microscope.

\section{Statistical analysis}

Data are reported as means \pm SE. Statistical analysis was performed using the SPSS version 10.0 software, with the $\chi^{2}$ test and Fisher's exact probability for numeration data. The independent sample group $t$-test and two-way analysis of variance (ANOVA) were used for measurement data. $\mathrm{P}<0.05$ was considered to be significant.

\section{RESULTS}

\section{Clinical classification and correlation between SODD expression in ALL cells}

Positive expression of SODD was located near the cell membrane in the cytoplasm, with a buffy granule, circular form, and sporadic distribution. In the negative controls, the cytoplasm and nuclei of the leukemic cells were dyed blue by hematoxylin, without buffy granules (Figure 1). The positive SODD expression in children with ALL is shown in Table 1. A higher expression of SODD was found in the ALL groups compared with the control group, especially the refractory/relapsed and HR groups. The positive expression rates of SODD and Bcl-2 in the refractory/relapsed group were 81.3 and $87.5 \%$, respectively. These were obviously higher than those in the initial treatment or CR groups. Among 55 cases with positive SODD expression, the positive Bcl-2 expression rate was $83.6 \%$. The positive SODD expression rate was $86.9 \%$ among 46 cases with positive Bcl-2 expression. These findings indicated a significant positive correlation between SODD and Bcl-2 expression based on the Pearson rank correlation analysis $(\mathrm{P}<0.01, \mathrm{r}=0.636)$. 


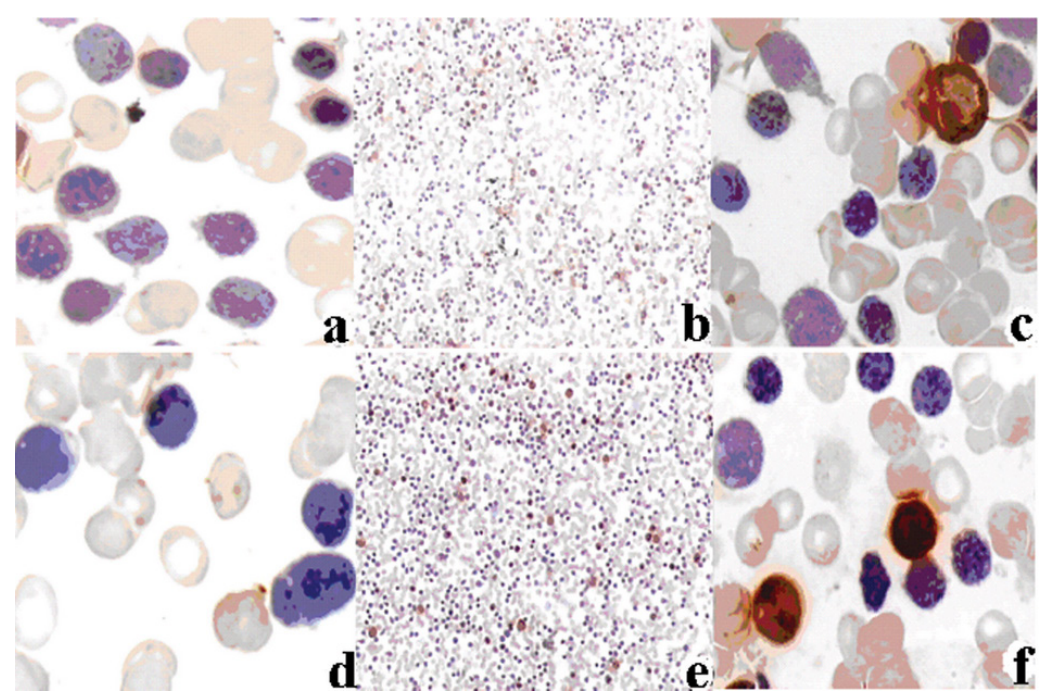

Figure 1. SODD expression in the bone marrow cells of children with ALL. The positive expression of SODD was located near the cell membrane in the cytoplasm, with a buffy granule, circular form, and sporadic distribution. a and $\mathbf{d}$ are the negative controls. $\mathbf{b}$ and $\mathbf{e}$ show the positive expressions of SODD and BcL-2 at $10 \mathrm{X}$ using the SABC method, respectively. $\mathbf{c}$ and $\mathbf{f}$ show the positive expressions of SODD and BcL-2 using the SABC method (100X), respectively.

Table 1. Positive expression of SODD in children with ALL.

\begin{tabular}{lcccc}
\hline Groups & Cases & SODD-positive expression $(\%)$ & P value & $\chi^{2}$ value \\
\hline Initial treatments & 51 & $29(56.9)$ & 0.0178 & 14.53 \\
Standard-risk & 32 & $14(43.8)$ & 0.0199 & 12.88 \\
High-risk & 19 & $15(78.9)$ & 0.0028 & 19.35 \\
Complete remission & 40 & $13(32.5)$ & 0.0393 & 15.28 \\
Refractory/relapsed & 16 & $13(81.3)$ & 0.0015 & 20.13 \\
Control & 26 & $2(7.7)$ & & \\
\hline
\end{tabular}

$\chi^{2}$ and $\mathrm{P}$ values were based on statistical comparison between the positive SODD expression in each ALL group and that in the normal control.

\section{Changes in SODD expression}

Commonly used chemotherapeutic drugs were applied on Jurkat cells. The cells in each sample group were harvested, and the total protein was extracted separately. Changes in SODD expression were detected by Western blot analysis. The results showed that the SODD expression decreased gradually with the time extension of VCR and with an increased concentration of PTX. Such effects were not found after the application of DNR, Dexa, MTX, Ara-c, or L-ASP. This finding indicated the specificity of VCR as time-dependent and of PTX as concentration-dependent in the down-regulation of SODD expression. SODD was not the target of DNR, Dexa, MTX, Ara-c, or L-ASP action (Figure 2). After 48 h of Jurkat cell transfected with SODD RNAi-interfering plasmids or SODD-cloning gene vectors, green fluorescence was observed under FCM at a transient transfection rate of $20 \%$. Transfected cells were collected, total protein was extracted, and changes in SODD expression were detected 
by Western blot analysis. The results showed that SODD expression in the cells transfected by interfering plasmids was lower than in the control group, whereas SODD expression in the cells transfected by SODD-cloning genes was higher (Figure 3).

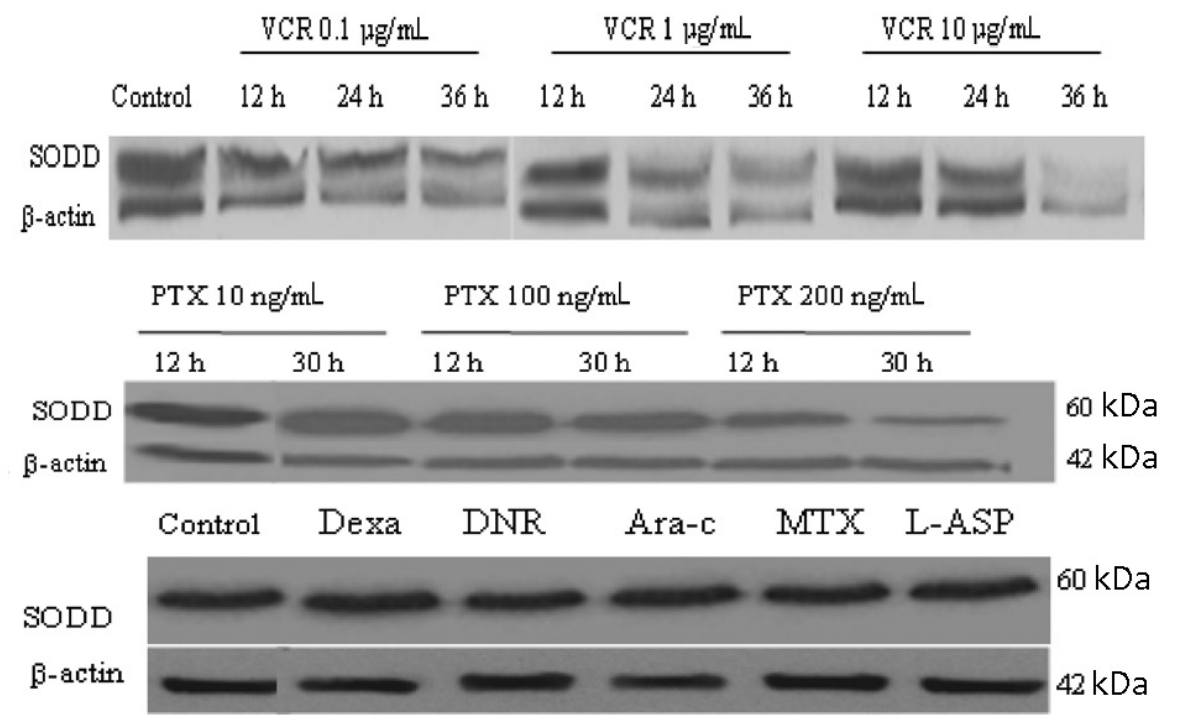

Figure 2. Changes in SODD expression in leukemic cells after VCR, PTX, DNR, Dexa, MTX, Ara-c, and L-ASP treatments.

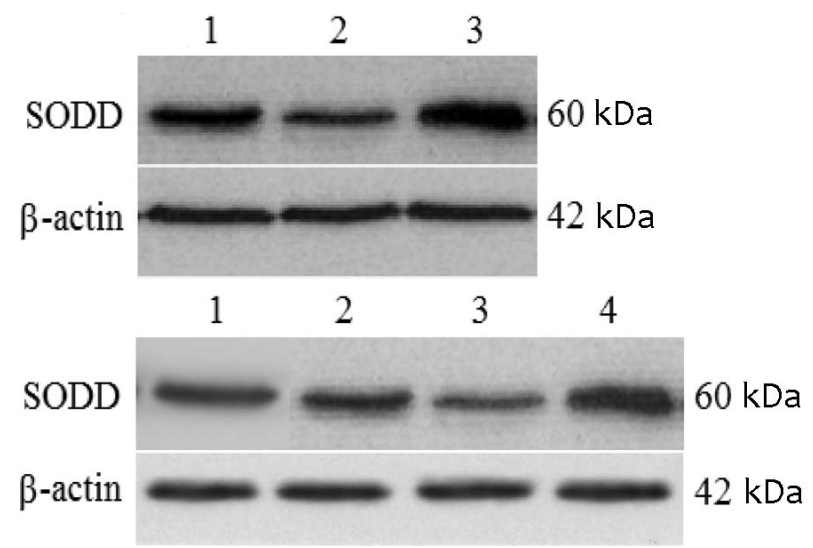

Figure 3. Changes in SODD expression in different transfection cell groups. Lane $1=$ control group; lane $2=$ group transfected with SODD-interfering micromolecular plasmids; lane $3=$ group transfected with SODD-RNAi interference; lane 4 = group transfected with SODD sub-cloning genes.

\section{Changes in Bcl-2 expression}

Changes in Bcl-2 expression in different drug-treated groups were detected by Western blot analysis. The results showed that both VCR and PTX could down-regulate 
Bcl-2 expression. However, VCR and PTX behaved differently. VCR down-regulated Bcl-2 expression in a time-dependent manner, whereas PTX down-regulated Bcl-2 expression in a concentration-dependent manner (Figure 4).

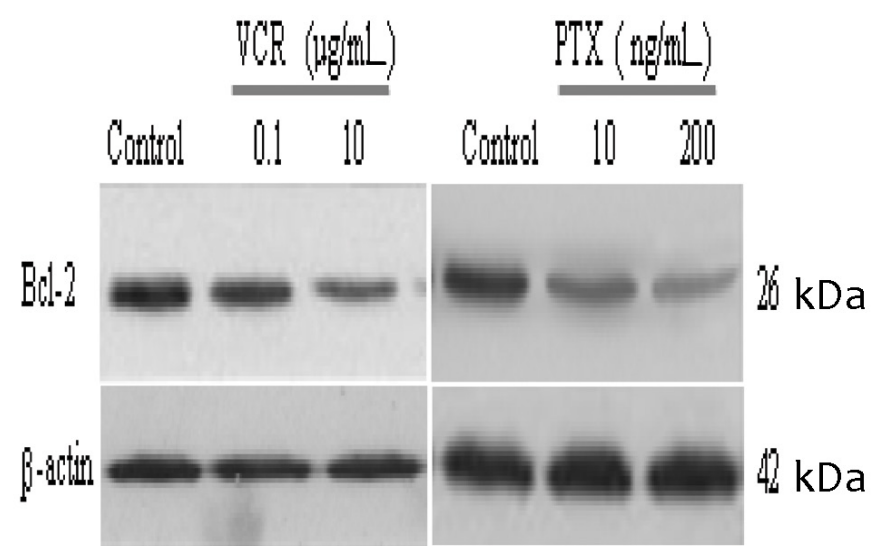

Figure 4. Changes in Bcl-2 expression in Jurkat cells induced by VCR and PTX.

\section{Apoptotic effects of SODD-targeting drugs}

Cell number was increased at the S and G2/M stages after treatment with VCR/PTX. Meanwhile, the hypodiploid apex (i.e., apoptotic peak) appeared before the G1 peak, indicating that VCR and PTX can induce the apoptosis of Jurkat cells in different groups effectively, with a low drug concentration for early apoptosis and a high concentration for advanced apoptosis and necrosis. The apoptosis rate of the group transfected with SODD RNAi-interfering plasmids was higher, whereas that of the group transfected with SODD-cloning genes was lower compared with the control group. These findings indicated a negative correlation with SODD expression. Therefore, a close correlation is suggested between SODD expression and the cellular response to drugs (Table 2).

Table 2. Apoptotic incidence of Jurkat cells treated by VCR and PTX respectively in different groups.

\begin{tabular}{lcc}
\hline Groups & Apoptotic incidence (\%) $12 \mathrm{~h}$ & Apoptotic incidence $(\%) 30 / 36 \mathrm{~h}$ \\
\hline Control $^{1}$ & 1.3 & 15.7 \\
VCR $^{2}$ & $64.4 / 28.6$ & $90.6 / 16.8$ \\
PTX $^{3}$ & $63.0 / 25.6$ & $89.1 / 15.3$ \\
PEGFP-C1-SODD-VCR $^{4}$ & $87.0 / 23.3$ & $99.0 / 19.6$ \\
PEGFP-C1-SODD-PTX $^{5}$ & $83.2 / 24.0$ & $97.4 / 17.1$ \\
RNAi-VCR & $53.0 / 22.5$ & $78.2 / 15.5$ \\
RNAi-PTX & $52.4 / 19.8$ & $71.4 / 18.8$ \\
\hline
\end{tabular}

1 vs 2 and 1 vs $3(\mathrm{P}<0.01) ; 2$ vs $3(\mathrm{P}>0.01) ; 1$ vs 4 and 1 vs $5(\mathrm{P}<0.01) ; 4$ vs $5(\mathrm{P}>0.01) ; 1$ vs 6 and 1 vs 7 ( $\mathrm{P}$ $<0.01) ; 6$ vs $7(\mathrm{P}>0.01) ; 2$ vs 4 and 4 vs $6(\mathrm{P}<0.01) ; 3$ vs 5 and 5 vs $7(\mathrm{P}<0.01)$.

\section{DISCUSSION}

SODD is a widely expressed cytoplasmic protein of 457-amino acid residues, with a molecular weight of $60 \mathrm{kDa}$. SODD was first isolated from a cDNA library by Jiang et 
al. (1999). As a member of the BAG protein family, SODD is also named BAG-4. SODD contains a sequence closely associated with cytotoxic activity, i.e., the DD. SODD exerts its biological function through its specific binding with DDs in the cytoplasmic regions of TNFRI, Bcl-2, DR3, and HSP/HSC70 (Briknarová et al., 2002). SODD integrates with the DD of TNFR-I to form an SODD-TNFR-I complex for the prevention of TNFR-I aggregation and the disruption of the TNFR-I signaling pathway. The stimulation of TNF- $\alpha$ can cause abscission of SODD from the SODD-TNFR-I complex, by which TNFR-I, in a monomeric state, interacts with other receptor-related proteins to maintain the signaling pathway. However, $10 \mathrm{~min}$ later, SODD reintegrates with the DD of TNFR-I to deactivate the TNFR-I signaling pathway and disrupt the apoptosis pathway (Annunziata et al., 2007). Currently, SODD has been identified as a negative-regulatory protein for the TNFR-I signaling pathway.

Studies have shown that cells with over-expression of SODD display insensitivity to the activation of TNFR-I cascades, NF- $\kappa B$, and JNK induced by TNF- $\alpha$. Continuous SODD expression inhibits the activation of NF-kB-dependent receptor genes and the apoptosis induced by TNF (Eichholtz-Wirth and Sagan, 2002; Takada et al., 2003). SODD showed a continuous high expression in human tumor cell lines such as U937, 293, HeLa, and Jurkat, among others (Eichholtz-Wirth et al., 2003). Over-expressed SODD can inhibit the apoptosis mediated by TNF/TNFR-I. This inhibition leads to a decreased sensitivity of tumor cells to the apoptosis mediated by death receptors and contributes further to drug resistance. Therefore, if the expression of SODD in cells is suppressed, the apoptotic sensitivity of cells can be restored (Miki and Eddy, 2002).

The Bcl-2 gene is the earliest discovered anti-apoptosis gene that can combine with SODD through the DD. Its coded product, the Bcl-2 protein, is also an anti-apoptosis factor (Letai, 2011; Maráz et al., 2011; Qiao et al., 2011). Studies of various solid tumors and adult leukemia showed that different tumor cell lines show different Bcl-2 expression. Cells with high Bcl-2 expression display apoptotic suppression as well as resistance to chemotherapeutic drugs (Yasmeen et al., 2011; Mendilcioglu et al., 2011). SODD binds specifically to Bcl-2 through the DD after its abscission from TNFR-I. However, the biological behavior at a later stage remains unclear. Both SODD and Bcl-2 are apoptosis-inhibitory factors.

ALL is one of the most common malignant tumors among children. At present, chemotherapy is the major treatment method for ALL patients. Relatively high remission and cure rates can be obtained clinically by chemotherapy, but $30 \%$ of cases still respond poorly. Among these cases, drug resistance is the main cause of treatment failure. Drug resistance is closely associated with severe genetic abnormalities, which is the key focus and major challenge in the study of leukemia to date (Pállinger et al., 2011). Our results showed overexpression of SODD and Bcl-2 in bone marrow cells in children with ALL, especially those with HR, drug resistance, and relapse. The positive expression of SODD and Bcl-2 in the HR group was higher compared with those in the standard-risk group. An obvious increase in expression can be found in the drug-resistance relapsed group. The suggested high expression of SODD and Bcl-2 serve as the indices of insensitivity to chemotherapy and poor prognosis, considering the close correlation between clinical classification and prognosis. Meanwhile, our results also showed that SODD expression in the CR group was lower than that in the initial treatment or refractory/relapsed group, yet higher than in the control group. Comparison of the expression before and after remission showed a down-regulation tendency of SODD expression as chemotherapy proceeded, which was consistent with previous studies (Tao et 
al., 2007a,b). This finding indicated the existence of a certain amount of abnormal cells with high SODD expression in CR patients, which resulted in subsequent relapse. Therefore, clinically, more attention should be given to the high expression of SODD, especially in children with remission. These findings do not only provide a reference for the use of an individualized treatment plan but also serve as bases for the evaluation of the treatment effects and prognosis of ALL children. Further, according to our study, the CR rate of children with positive SODD and Bcl-2 expression was significantly lower than that of children with negative SODD/Bcl-2 or SODD and Bcl-2 expression. These data suggest that both SODD and Bcl-2 have close correlation with the initiation, development, and prognosis of ALL and play a synergistic effect on ALL development. Therefore, the suppression of their expressions is crucial for improving the treatment of leukemia.

In the present study, screening of chemotherapeutics that can specifically down-regulate SODD expression was conducted for common drugs using human acute T-ALL Jurkat cells as the experimental model. Western blot analysis showed that drugs targeting microtubules such as VCR, PTX, and Colchicin can down-regulate SODD expression effectively and lead to leukemic cell apoptosis in a time- and dose-dependent manner. Simultaneously, these chemotherapeutics can also down-regulate Bcl-2 expression in leukemic cells. However, such an effect on SODD expression was not found among other cytotoxic drugs commonly used in the combined chemotherapy of ALL such as DNR, Ara-c, and VP-16, among others. These results suggested that SODD may just be the specific target of drugs targeted at microtubules, and their genetic regulation may represent a special tumor cell apoptosis signaling pathway induced by drugs targeted at microtubules.

The high expression of SODD played a noticeable role in the escape from apoptosis induced by chemotherapeutics for leukemic cells (Stanton et al., 2011; Bounoutas et al., 2011). As the blockage of SODD expression in leukemic cells would be conducive to the restoration of the sensitivity of tumor cells during chemotherapy and to the increase in survival rate, SODD-targeting gene therapy can be of great potential value (Chen et al., 2008, 2011; Ito et al., 2011). Gene therapy is a technique for correcting defective genes or abnormal gene expression responsible for disease development. It is implemented by introducing genes into the targeted cells of the host and making these genes part of the host genetic material through the combination of genes (Tadin-Strapps et al., 2011). In our study, after shRNA-S (an SODDspecific shRNA eukaryotic expression vector) had been constructed, it was inserted into human acute T-ALL Jurkat cells through Lipofectamine transfection (Premsrirut et al., 2011; Nong et al., 2011). Meanwhile, to disprove that a high SODD expression led to the escape of leukemic cells from apoptosis and further to the insensitivity to drugs, an SODD gene was cloned and then transfected into Jurkat cells (Wang et al., 2010; Shen et al., 2011). Positive clones were obtained in cells under the selection of G418. Strong fluorescence was observed under FCM, which indicates that the introduced recombinant genes (by plasmid transfection) had been inserted successfully into the targeted cells and expressed continuously (Yang et al., 2010). Immunohistochemistry showed that SODD expression of the group stably transfected with RNAi-interfering plasmids was lower, whereas that of the group transfected with SODDcloning genes was higher compared with that of the control group. FCM analysis showed that the sensitivity of the group with decreased SODD protein expression to chemotherapeutics was increased. The cell growth-inhibitory rate and apoptotic effect by chemotherapeutics were significantly lower than those of the non-transfected groups. Those in the group with SODD- 
cloning genes were lower. In contrast, slower cell growth was observed in the group transfected with interfering plasmid. These results suggest that the SODD gene plays important roles in the process of Jurkat cell proliferation and apoptosis. The suppression of SODD expression assists substantially in the increased sensitivity of Jurkat cells to chemotherapeutics.

To some degree, a high expression of SODD can increase the tolerability of Jurkat cells to chemotherapeutics. Therefore, if the clinical significance of SODD and Bcl-2 expression in leukemia is identified from the perspective of molecular mechanism, the key point for the reversal of the drug resistance of leukemic cells can be determined, and the curative effect on refractory leukemia can be increased. This will be facilitated by the elucidation of the molecular mechanism of microtubule-targeting drugs in down-regulation of SODD expression and the clarification of the correlation between SODD and Bcl-2 as well as their pathways.

\section{Conflicts of interest}

The authors declare no conflict of interest.

\section{REFERENCES}

Annunziata CM, Kleinberg L, Davidson B, Berner A, et al. (2007). BAG-4/SODD and associated antiapoptotic proteins are linked to aggressiveness of epithelial ovarian cancer. Clin. Cancer Res. 13: 6585-6592.

Bounoutas A, Kratz J, Emtage L, Ma C, et al. (2011). Microtubule depolymerization in Caenorhabditis elegans touch receptor neurons reduces gene expression through a 38 MAPK pathway. Proc. Natl. Acad. Sci. U. S. A. 108: 3982-3987.

Briknarová K, Takayama S, Homma S, Baker K, et al. (2002). BAG4/SODD protein contains a short BAG domain. $J$. Biol. Chem. 277: 31172-31178.

Chen SL, Dai SM, Lu KH and Chang C (2008). Female-specific doublesex dsRNA interrupts yolk protein gene expression and reproductive ability in oriental fruit fly, Bactrocera dorsalis (Hendel). Insect. Biochem. Mol. Biol. 38: 155-165.

Chen SL, Lu KH, Dai SM, Li CH, et al. (2011). Display female-specific doublesex RNA interference in early generations of transformed oriental fruit fly, Bactrocera dorsalis (Hendel). Pest. Manag. Sci. 67: 466-473.

Cwiklińska M, Balwierz W and Stanuch H (2010). Clinical tolerance of high-dose methotrexate used in consolidation therapy in children with acute lymphoblastic leukemia. Przegl. Lek. 67: 355-360.

Czech MP, Aouadi M and Tesz GJ (2011). RNAi-based therapeutic strategies for metabolic disease. Nat. Rev. Endocrinol. 7: 473-484.

Dłuzniewska A, Balwierz W, Balcerska A, Chybicka A, et al. (2010). Treatment failure in children with acute myelocytic leukemia: over 25-year experience of Polish Pediatric Leukemia/Lymphoma Study Group with four consecutive unified treatment protocols for childhood acute myelocytic leukemia. Przegl. Lek. 67: 366-370.

Eichholtz-Wirth H and Sagan D (2002). Altered signaling of TNFalpha-TNFR1 and SODD/BAG4 is responsible for radioresistance in human HT-R15 cells. Anticancer Res. 22: 235-240.

Eichholtz-Wirth H, Fritz E and Wolz L (2003). Overexpression of the 'silencer of death domain', SODD/BAG-4, modulates both T. Cancer Lett. 194: 81-89.

Elstrand MB, Kleinberg L, Kohn EC, Trope CG, et al. (2009). Expression and clinical role of antiapoptotic proteins of the bag, heat shock, and Bcl-2 families in effusions, primary tumors, and solid metastases in ovarian carcinoma. Int. J. Gynecol. Pathol. 28: 211-221.

Ito H, Gaubert H, Bucher E, Mirouze M, et al. (2011). An siRNA pathway prevents transgenerational retrotransposition in plants subjected to stress. Nature 472: 115-119.

Jiang Y, Woronicz JD, Liu W and Goeddel DV (1999). Prevention of constitutive TNF receptor 1 signaling by silencer of death domains. Science 283: 543-546.

Letai A (2011). A new face of BCL-2 inhibition in CLL. Blood 117: 2750-2751.

Maráz A, Furak J, Palfoldi R, Eller J, et al. (2011). Roles of BCL-2 and MDR1 expression in the efficacy of paclitaxelbased lung cancer chemoradiation. Anticancer Res. 31: 1431-1436.

Mendilcioglu I, Karaveli S, Erdogan G, Simsek M, et al. (2011). Apoptosis and expression of Bcl-2, Bax, p53, caspase-3, and Fas, Fas ligand in placentas complicated by preeclampsia. Clin. Exp. Obstet. Gynecol. 38: 38-42. 
Miki K and Eddy EM (2002). Tumor necrosis factor receptor 1 is an ATPase regulated by silencer of death domain. Mol. Cell Biol. 22: 2536-2543.

Nong XL, Li H, Xia Y, Li Y, et al. (2011). In vivo short hairpin RNA interference of vascular endothelial growth factor on drug-resistant tongue cancer cells. Zhonghua Kou Qiang. Yi Xue Za Zhi 46: 15-19.

Pállinger E, Horvath Z, Csoka M, Kovacs GT, et al. (2011). Decreased hormone content of immune cells in children during acute lymphocytic leukemia (ALL) - effect of treatment. Acta Microbiol. Immunol. Hung. 58: 41-50.

Premsrirut PK, Dow LE, Kim SY, Camiolo M, et al. (2011). A rapid and scalable system for studying gene function in mice using conditional RNA interference. Cell 145: 145-158.

Qiao WL, Wang GM, Shi Y, Wu JX, et al. (2011). Differential expression of Bcl-2 and Bax during gastric ischemiareperfusion of rats. World J. Gastroenterol. 17: 1718-1724.

Shen HL, Fang LL, Chen C, Fang XJ, et al. (2011). VEGF shRNA enhances the sensitivity of multidrug-resistant leukemia cells to anticancer agent. Zhongguo Shi Yan Xue Ye. Xue Za Zhi 19: 34-39.

Stanton RA, Gernert KM, Nettles JH and Aneja R (2011). Drugs that target dynamic microtubules: a new molecular perspective. Med. Res. Rev. 31: 443-481.

Tadin-Strapps M, Peterson LB, Cumiskey AM, Rosa RL, et al. (2011). siRNA-induced liver ApoB knockdown lowers serum LDL-cholesterol in a mouse model with human-like serum lipids. J. Lipid Res. 52: 1084-1097.

Takada H, Chen NJ, Mirtsos C, Suzuki S, et al. (2003). Role of SODD in regulation of tumor necrosis factor responses. Mol. Cell Biol. 23: 4026-4033.

Tao H, Hu Q, Fang J, Liu A, et al. (2007a). Expression of SODD and P65 in ALL of children and its relationship with chemotherapeutic drugs. J. Huazhong. Univ. Sci. Technolog. Med. Sci. 27: 326-329.

Tao HF, Hu Q, Fang JL, Liu AG, et al. (2007b). Effects of SODD and survivin on leukemia cell apoptosis induced by chemotherapeutic drugs. Zhongguo Shi Yan Xue Ye. Xue Za Zhi 15: 501-505.

Wang TY, Feng SQ, Zhang ZX, Shi XD, et al. (2010). Suppression of survivin gene in leukemia cells by small interfering RNA. Zhonghua Er. Ke. Za Zhi 48: 843-847.

Xi G, Hu X, Wu B, Jiang H, et al. (2011). Autophagy inhibition promotes paclitaxel-induced apoptosis in cancer cells. Cancer Lett. 307: 141-148.

Yang J, Ikezoe T, Nishioka C, Furihata M, et al. (2010). AZ960, a novel Jak2 inhibitor, induces growth arrest and apoptosis in adult T-cell leukemia cells. Mol. Cancer Ther. 9: 3386-3395.

Yasmeen A, Beauchamp MC, Piura E, Segal E, et al. (2011). Induction of apoptosis by metformin in epithelial ovarian cancer: involvement of the Bcl-2 family proteins. Gynecol. Oncol. 121: 492-498. 